\title{
Oralmotor slowing in multiple sclerosis: Relationship to neuropsychological tasks requiring an oral response
}

\author{
PETER A. ARNETT, ${ }^{1}$ MEGAN M. SMITH,${ }^{1}$ FIONA H. BARWICK, ${ }^{1}$ RALPH H.B. BENEDICT, ${ }^{2}$ \\ AND BRIAN P. AHLSTROM ${ }^{3}$ \\ ${ }^{1}$ Psychology Department, The Pennsylvania State University, University Park, Pennsylvania \\ ${ }^{2}$ Department of Neurology, State University of New York at Buffalo, and the Jacobs Neurological Institute, Buffalo, New York \\ ${ }^{3}$ Private Neurology Practice, Johnstown, Pennsylvania \\ (Received November 12, 2006; Final Revision January 2, 2008; Accepted January 2, 2008)
}

\begin{abstract}
Although most neuropsychological batteries used with multiple sclerosis (MS) patients now exclude tests that require significant motor writing or manual manipulation speed, many of the most sensitive commonly used cognitive tests nonetheless require some type of rapid oral motor response. The aim of this study is to examine the extent to which primary oral motor articulation speed problems of individuals with MS contribute to performance and group differences on neuropsychological tasks requiring a rapid spoken response. Fifty MS patients and 50 healthy controls were administered the PASAT, COWAT, Animal Naming, and SDMT tests, in addition to a measure of rudimentary oral motor speed known as the maximum repetition rate of syllables and multisyllabic combinations (MRR) task. Regression analyses revealed that the amount of variance accounted for by the group (MS-Control) variable was reduced the following amounts for the tasks when the MRR was entered before the group variable: SDMT, $10 \%$ to 6\%; PASAT, $4 \%$ to 2\%; COWAT, 5\% to 2\%; Animal Naming, $11 \%$ to $7 \%$. Our data suggest that rudimentary oral motor speed is slowed in MS patients and makes an important contribution to group differences in performance on commonly used neuropsychological tasks requiring a rapid spoken response.

(JINS, 2008, 14, 454-462.)
\end{abstract}

Keywords: Multiple sclerosis, Neuropsychological functioning, Cognitive functioning, Oral motor speed, Processing speed

\section{INTRODUCTION}

It has long been appreciated that input and output problems can influence performance on neuropsychological tests (Weintraub \& Mesulam, 1985). A central goal in clinical neuropsychology is to draw conclusions about the nature of cognitive difficulties with our tests. To accomplish this, however, the contribution of possible input or output problems must first be addressed. Deficits in response output (typically written/manual or spoken responses) or input (usually visual or auditory) systems can result in impaired neuropsychological test performance and make it difficult to draw conclusions about central or cognitive processing.

Correspondence and reprint requests to: Peter Arnett, Ph.D., Associate Professor, Penn State University, Psychology Department, 522 Bruce V. Moore Building, College of the Liberal Arts, University Park, PA. E-mail: paa6@psu.edu
Most neuropsychological tests recommended for the neuropsychological assessment of multiple sclerosis (MS) patients require a motor response. Although many batteries used with MS patients now exclude tests that require writing or drawing, thus circumventing one potential "output" influence, many of the most sensitive tests require some type of rapid oral response. For example, the one cognitive test included as part of the MS Functional Composite (Fischer et al., 1999), the Paced Auditory Serial Addition Test (PASAT), requires a rapid oral response. Other clinical neuropsychological tests requiring rapid oral responses that are commonly recommended for use in MS include the Controlled Oral Word Association Test (COWAT; Benton \& Hamsher, 1989), and the oral form of the Symbol Digit Modalities Test (SDMT; A. Smith, 1982). Because of their sensitivity to cognitive dysfunction in MS (Henry \& Beatty, 2006), both tests (and the PASAT) are recommended for use as part of Rao's Brief Repeatable Battery (BRB; S.M. 
Rao, and the Cognitive Function Study Group of the National Multiple Sclerosis Society, 1990), and the Minimal Assessment of Cognitive Functioning in MS (MACFIMS; Benedict et al., 2002). To date, however, the extent to which more primary problems with rapid articulation speed might influence performance on tests like these has only been minimally examined. If primary oral motor speed is slowed in MS patients, it may unduly contribute to their performance on these tests, inflating their deficit. The primary goal of the present study was to systematically evaluate this issue using performance-based measures of rudimentary oral motor speed in an MS sample.

As noted by Smith and Arnett (2007), Charcot observed that dysarthria (or "scanning speech") was one of the three characteristic neurological symptoms of MS, with the other two being intention tremor and nystagmus (Charcot, 1877; Darley et al., 1972). He noted its presence in 22 of the 23 cases he had examined and observed that, "... the words are as if measured or scanned; there is a pause after every syllable, and the syllables themselves are pronounced slowly" (1877, p. 192). Dysarthria is found in $40 \%$ to $55 \%$ of MS patients (Darley et al., 1972; Hartelius et al., 2000a; Hartelius et al., 2000b) and is more common in progressive MS course types (Hartelius et al., 2000a).

Similar to dysarthria, cognitive deficits are a common and prominent feature of MS. Research suggests that approximately $50 \%$ of MS patients are impaired on NP testing (Amato et al., 2006; Arnett, 2003; Benedict et al., 2006; Brass et al., 2006; Brassington \& Marsh, 1998; Jonsson et al., 2006; Rao et al., 1991). As noted earlier, many of the tasks that are most sensitive to cognitive dysfunction in MS require the patient to make a rapid oral motor response. To date, however, only one study has empirically examined the relationship between performance on these tasks and dysarthria. Smith and Arnett (2007) used examiner ratings of dysarthria (1-4 scale) and assessed the relationship of these ratings to performance on three tasks requiring a rapid oral motor response: The SDMT (A. Smith, 1982), the COWAT (Benton \& Hamsher, 1989), and the Visual Elevator subtest from the Test of Everyday Attention (Robertson et al., 1994). In this study, significantly more patients than healthy controls displayed dysarthria, and patients showed significantly worse performance than controls on the SDMT and Visual Elevator tests, even after controlling for relevant demographic and illness variables. In additional regression analyses where dysarthria ratings were entered in before the group variable, group differences on the Visual Elevator test were no longer statistically significant and the group effect for the SDMT, whereas still significant, was reduced by about one-third.

The results of Smith and Arnett (2007) suggest that slowed speech in MS patients contributes significantly to performance on at least some higher level neuropsychological tasks in MS patients. The data from this study also indicate that differential slowing of speech by MS patients contributes significantly to the group differences observed when patients are compared with healthy controls, at least for the
SDMT and Visual Elevator tasks. One limitation to this study, however, is the use of subjective ratings based on an unvalidated measure of dysarthria. In the present study we endeavored to improve upon prior research by widening the scope of cognitive testing and using an objective measure of oral motor speed known as the Maximum Repetition Rate of Syllables and Multisyllabic Combinations task (MRR; Kent et al., 1987), which has well-established reliability (Kreul, 1972) and validity (Dworkin et al., 1980; Portnoy \& Aronson, 1982; Tatsumi et al., 1979; Ziegler \& Wessel, 1996).

With these considerations in mind, the present study was designed to evaluate the extent to which primary oral motor articulation speed problems of individuals with MS contribute to performance and group differences on four tasks that have been shown to be among the most sensitive to cognitive dysfunction in MS and that also require a rapid oral motor response: The PASAT, SDMT, COWAT, and Animal Naming tests. Our study had the following hypotheses: (1) compared with healthy controls, MS patients will perform significantly worse on a performance-based measure of oral motor speed and on the four neuropsychological tasks; (2) performance on the oral motor speed task will be positively correlated with performance on the four neuropsychological tasks in MS patients and controls; and (3) after removing variance caused by oral motor speed, group differences between MS patients and healthy controls on the four neuropsychological tasks will be reduced. Finally, to examine the discriminant validity of the oral motor speed task, two neuropsychological tasks not requiring a rapid oral motor response were included, the BVMT-R and the CVLT-II.

\section{METHODS}

\section{Participants}

\section{MS group}

The MS group consisted of 51 individuals with MS. One patient could not be included in the analyses because data from the oral motor task (described later) were lost because of experimenter error. Thus, 50 patients ( 40 women, 10 men) were included in most analyses. Prior to being scheduled for testing, participants were administered a structured telephone screening interview to determine their eligibility for the project by evaluating the following inclusionary criteria: (a) No history of alcohol/drug abuse or nervous system disorder other than MS; (b) no sensory impairments that might interfere significantly with cognitive testing; (c) no developmental history of a learning disability or attentiondeficit/hyperactivity disorder; (d) no medical condition other than MS that could substantially affect cognition or motor function; (e) no relapse and/or corticosteriod use within four weeks of assessment; or (f) absence of severe physical/ neurological impairment that would make testing impossible. 
Multiple sclerosis diagnoses, based on the McDonald et al. (2001) criteria, were confirmed by a board-certified neurologist (in most cases, by the fifth author, B.A.) who also assessed disease course based on Lublin and Reingold (1996) criteria. There were 29 patients with a relapsing-remitting course, 14 with secondary progressive, four with primary progressive, and three with progressive relapsing. Duration of illness from symptom onset and from diagnosis, as well as neurological disability (Kurtzke Expanded Disability Status Scale [EDSS], Kurtzke, 1983), were also rated. None of the patients included in the current study were experiencing a clinical exacerbation at the time of the evaluation. In return for their participation, all participants were paid $\$ 100$ and provided with a written neuropsychological screening evaluation and verbal feedback.

\section{Healthy control group}

Fifty-one neurologically healthy community-based controls were recruited. One control could not be included in the analyses because data from the oral motor task (described later) were lost because of experimenter error. Thus, 50 controls (42 women and 8 men) were included in most analyses. An attempt was made to match controls with the MS participants on demographic features (i.e., age, education, and gender) as closely as possible. The same relevant inclusionary criteria employed with the MS patients were used. Control participants were recruited by asking MS participants to recommend a friend, by posting advertisements in public places in Central Pennsylvania, and also via a university newswire. Controls were also paid $\$ 100$ for their participation. All participants gave informed consent according to institutional guidelines and were treated in accordance with the ethical standards of the American Psychological Association. The study was approved by the Institutional Review Board at Penn State University. Participant characteristics are outlined in Table 1.

\section{Procedure}

Participants in both groups completed the neuropsychological tests in addition to a larger battery of neuropsychological tests as part of an ongoing longitudinal study of cognitive and emotional changes in MS. A psychosocial interview was conducted prior to the cognitive assessment in order to obtain basic demographic information including education and age. Cognitive tasks, the oral motor tasks, and the depression and fatigue measures were administered on the same day. Two test orders were given. In one, the MRR was administered near the beginning of the test battery and for the other it was administered toward the end.

\section{Measures}

\section{Maximum Repetition Rate of Syllables and Multisyllabic Combinations (MRR;}

Kent et al., 1987)

The MRR is one of the most commonly used tasks to measure oral motor speed in the speech and language literature. It has been recommended for use as a control for oral motor slowing deficits in MS as part of the MACFIMS (Benedict et al., 2002), but has not yet been empirically examined for this purpose. Kent and colleagues (1987) note, in their review of tests of speech production, that "... the monosyllabic triad [pa], [ta], [ka] has become a clinical standard ..." (p. 379) for which the greatest amount of normative data is available. The task requires examinees to repeat these syllables as quickly as they can in one good breath and they are typically assessed for six seconds. The syllables "pa," "ta," and "ka" are repeated in separate trials, and then a final trial requiring the repetition of "pa-ta-ka" in sequence is conducted. Data for each of the four tasks are converted to syllables/second.

Table 1. Characteristics and $t$-values for multiple sclerosis and healthy control participants

\begin{tabular}{|c|c|c|c|c|c|c|}
\hline \multirow[t]{2}{*}{$\begin{array}{l}\text { Variable } \\
n\end{array}$} & \multicolumn{2}{|c|}{$\begin{array}{c}M S \\
50\end{array}$} & \multicolumn{2}{|c|}{$\begin{array}{l}\text { Healthy Controls } \\
50\end{array}$} & \multirow[b]{2}{*}{$t(98)$} & \multirow[b]{2}{*}{$P$} \\
\hline & $M$ & $S D$ & $M$ & $S D$ & & \\
\hline Education (years) & 14.7 & 2.1 & 15.7 & 2.4 & -2.21 & $<.05$ \\
\hline Age & 51.9 & 9.3 & 45.8 & 10.5 & 3.09 & $<.005$ \\
\hline BDI-II & 9.9 & 6.6 & 3.4 & 4.3 & 5.82 & $<.001$ \\
\hline FSS & 5.4 & 1.3 & 2.8 & 1.0 & 10.72 & $<.001$ \\
\hline EDSS & 4.0 & 2.2 & & & & \\
\hline Symptom Duration (years) & 18.0 & 9.3 & & & & \\
\hline Diagnosis Duration (Years) & 14.5 & 8.7 & & & & \\
\hline
\end{tabular}

Note. BDI-II = Beck Depression Inventory-II. EDSS = Expanded Disability Status Scale (Kurtzke, 1983); FSS = Fatigue Severity Scale (Krupp et al., 1989). Symptom and Diagnosis Duration are indicated in years. 


\section{Cognitive measures requiring a rapid oral response}

With the exception of animal naming, all of the following tasks have been recommended for use with MS patients as part of Rao's BRB (S. M. Rao, and the Cognitive Function Study Group of the National Multiple Sclerosis Society, 1990) and the MACFIMS (Benedict et al., 2002).

Paced Auditory Serial Addition Test (PASAT; S.M. Rao and the Cognitive Function Study Group of the National Multiple Sclerosis Society, 1990)

The PASAT is conceptualized as a measure of sustained attention, working memory, and speeded information processing (Lezak et al., 2005). We used the $3 \mathrm{~s}$ and $2 \mathrm{~s}$ versions of the PASAT described as part of Rao's BRB (S. M. Rao, and the Cognitive Function Study Group of the National Multiple Sclerosis Society, 1990). The PASAT involves single-digit numbers being presented auditorily via a compact disc player every three or every two seconds depending upon the version. Participants are instructed to add each successive digit to the one presented immediately before it. Number of correct additions out of 60 possible was used as the dependent variable for each version of the task and these were then combined into one index for data analysis.

\section{Symbol Digit Modalities Test (SDMT),}

\section{Oral Form (A. Smith, 1982)}

The SDMT is a measure of complex scanning and visual tracking (Lezak et al., 2005) as well as working memory and information processing speed. It reverses the presentation of Wechsler's (1997) Digit Symbol test so that the symbols are printed and the numbers are spoken aloud. Rao's $\mathrm{BRB}$ oral adaptation of the test was used and total correct in 90 seconds was the dependent measure.

\section{Controlled Oral Word Association Test (COWAT; Benton \& Hamsher, 1989)}

The COWAT, a measure of verbal fluency, involves the rapid oral generation of words from a phonemic cue. The CFL version was used in the present examination. The dependent measure from this test was the number of words generated in three 60 -second trials.

\section{Animal Naming Test (Strauss et al., 2006)}

The Animal Naming Test also measures verbal fluency but uses a semantic cue. Examinees must name as many animals as possible in one minute. The dependent measure was the number of animal words generated.

\section{Cognitive measures not requiring a rapid oral response}

Both of the following tasks have been recommended for use with MS patients as part of the MACFIMS (Benedict et al., 2002). These were included so that the discriminant validity of the MRR task could be evaluated.

\section{California Verbal Learning Test, 2nd edition (CVLT-II; Delis et al., 2000)}

The CVLT-II measures one's ability to learn and remember a list of 16 words, which have been verbally presented. The dependent measure was the $t$-score for total recall on the primary immediate recall index, Trials $1-5$ Recall.

\section{Brief Visual Memory Test-Revised (BVMT-R; Benedict, 1997)}

The BVMT-R measures visual memory and involves the presentation of the same six designs over three trials followed by delayed recall. We used total recall across the three immediate recall trials as the dependent measure.

\section{Measures to assess secondary influences}

Numerous studies have shown that MS patients consistently report higher levels of depression and fatigue when compared with healthy controls (Arnett, 2003; Brassington \& Marsh, 1998; Fischer et al., 1994; Tröster \& Arnett, 2006). We attempted to control for these factors with two selfreport tests, the Beck Depression Inventory-2nd edition (BDI-II; Beck et al., 1996) and the Fatigue Severity Scale (FSS; Krupp et al., 1989). The BDI-II is one of the most commonly used self-report measures of depression. It consists of 21 items on which participants rate themselves on a 0-3 scale. Higher scores reflect greater depression. The FSS is one of the most commonly used measures of fatigue in MS. It consists of nine items that examinees rate on a 1-7 Likert scale ( 1 = "Strongly Disagree," 7 = "Strongly Agree"). Higher scores represent greater levels of fatigue. The dependent variable was the mean score per scale item.

\section{Data Analytic Strategy}

Group differences for most illness/demographic variables were determined with $t$-tests. If group differences were found, these variables were then correlated with the dependent variables used in the analyses. Any variables that were significantly correlated with a dependent variable were subsequently controlled for in the statistical analyses (described later). A chi-square analysis was used to evaluate group differences in sex. Regression analyses were conducted to assess the extent to which group differences in oral motor speed contributed to group differences on the cognitive measures requiring a rapid oral response. To accomplish this, we conducted standard regression analyses where variables were entered into the analysis in two pre-determined orders for each cognitive measure (e.g., SDMT, PASAT) serving as a dependent variable. In one instance we entered the group variable (MS-Control) first followed by the MRR mean. In the other instance we entered 
the MRR mean first and then the group variable. By comparing the amount of variance accounted for by the group variable before and after the MRR task was entered, we could directly estimate how much the group effect was reduced after the influence of the MRR variable was accounted for.

\section{RESULTS}

\section{Preliminary Data Evaluation}

Comparison of the groups on demographic and illness variables (detailed in Table 1) revealed that the MS group was significantly older and had significantly less education than controls. As a result, when these variables were significantly $(p<.05)$ correlated with any dependent variables in the analyses, they were controlled for appropriately, as described later. Possible order effects for the MRR task were analyzed using a 2 (order) $\times 2$ (group) ANOVA. There was a significant main effect for order $\left(F_{(1,96)}=4.08\right.$, $p<.05)$ such that participants receiving the task near the beginning of the test day performed more quickly; however, this was true across MS and control groups. The group $\times$ order interaction was negligible $\left(F_{(1,96)}<1.0\right.$, $p>.99$ ), so order effects were not controlled for in the primary analyses.

One participant (an MS patient) was unable to complete the 2s PASAT. Following a strategy taken in our prior work (Arnett et al., 1999) and by Dikmen and colleagues (Dikmen et al., 1995), we assigned a value one point below the lowest point of the distribution for the 2s PASAT for this participant. This approach did not significantly change the results, but we decided on it in the interest of providing the most complete picture of our sample.

\section{Primary Analyses}

A multivariate ANOVA was conducted to compare groups on the MRR tasks. The "pa," "ta," "ka," and "pa-ta-ka" tasks were the four dependent variables, and control-MS group was the between groups factor. Because education was significantly correlated with the MRR tasks, it was included as a covariate in the analysis. As shown in Table 2, the multivariate effect revealed that, overall, the MS group was significantly slower than the control group on the MRR tasks $\left(F_{(4,94)}=2.64, p<.05\right)$. Univariate tests revealed that MS patients were significantly slower than controls on both the "pa" $(p<.02)$ and "ka" $(p<.005)$ tests, with statistical trends $(p<.10)$ in the same direction for the "ta" and "pa-ta-ka" tests.

A second multivariate ANOVA was conducted to compare groups on the neuropsychological tasks. The combined PASAT, COWAT, Animal Naming, and SDMT tests were the four dependent variables, and control-MS group was the between groups factor. Because age was significantly correlated with three of the tasks and education with two, both variables were included as covariates in the analysis. As shown in Table 2, the multivariate effect revealed that, overall, the MS group performed significantly worse than the control group on these speeded neuropsychologi-

Table 2. MRR and neuropsychological test scores for multiple sclerosis and healthy control participants

\begin{tabular}{|c|c|c|c|c|c|c|}
\hline \multirow[t]{2}{*}{$\begin{array}{l}\text { Variable } \\
n\end{array}$} & \multicolumn{2}{|c|}{$\begin{array}{c}M S \\
50\end{array}$} & \multicolumn{2}{|c|}{$\begin{array}{l}\text { Healthy Controls } \\
\qquad 50\end{array}$} & & \multirow[b]{2}{*}{$P$} \\
\hline & $M$ & $S D$ & $M$ & $S D$ & & \\
\hline \multirow[t]{2}{*}{ Multivariate F $(4,94)$ for MRR } & & & & & 2.64 & $<.05$ \\
\hline & & & & & $\begin{array}{l}\text { Univariate } \\
\mathrm{F}(1,97)\end{array}$ & \\
\hline $\operatorname{MRR}(\mathrm{pa})$ & 4.72 & 0.97 & 5.18 & 0.85 & 6.01 & $<.02$ \\
\hline $\operatorname{MRR}(\mathrm{ta})$ & 4.66 & 1.01 & 5.08 & 0.91 & 3.78 & $<.06$ \\
\hline MRR (ka) & 4.30 & 0.96 & 4.83 & 0.86 & 7.17 & $<.005$ \\
\hline MRR (pa-ta-ka) & 4.59 & 1.23 & 5.14 & 1.08 & 4.26 & $<.08$ \\
\hline \multirow{2}{*}{$\begin{array}{l}\text { Multivariate F }(4,93) \text { for } \\
\text { neuropsychological tasks }\end{array}$} & & & & & 4.52 & $<.005$ \\
\hline & & & & & $\begin{array}{l}\text { Univariate } \\
\mathrm{F}(1,96)\end{array}$ & \\
\hline PASAT $(2 s+3 s)$ & 72.2 & 21.5 & 86.2 & 21.9 & 4.83 & $<.05$ \\
\hline SDMT (Oral) & 54.6 & 13.2 & 66.7 & 12.2 & 12.87 & $<.001$ \\
\hline Animal Naming & 20.2 & 5.1 & 24.8 & 4.7 & 13.35 & $<.001$ \\
\hline COWAT & 40.1 & 11.1 & 45.3 & 11.7 & 3.47 & $<.07$ \\
\hline
\end{tabular}

Note MRR = Maximum Repetition Rate of Syllables and Multisyllabic Combinations task (Kent et al., 1987); PASAT = Paced Auditory Serial Addition Test (S. M. Rao, and the Cognitive Function Study Group of the National Multiple Sclerosis Society, 1990); COWAT $=$ Controlled Oral Word Association Test (Benton \& Hamsher, 1989); SDMT = Symbol Digit Modalities Test (A. Smith, 1982). All MRR values in syllables per second. 
cal tasks $\left(F_{(4,93)}=4.52, p<.005\right)$. Univariate tests revealed that MS patients performed significantly worse than controls on the PASAT $(p<.05)$, SDMT $(p=.001)$, and Animal Naming $(p<.001)$, with a statistical trend $(p<.07)$ in the same direction for the COWAT.

Next, regression analyses were conducted to evaluate the contribution of group differences in oral motor speed to group differences on the cognitive measures requiring a rapid oral response. ${ }^{1}$ Because the MRR tasks were all highly correlated ( $r$ range from .58 to .83 ), one mean index score for all four tasks was used in the analyses. We also combined scores to create a more stable index of oral motor speed that is less likely to be influenced by extreme scores, and because we wished to keep the number of analyses we conducted to a reasonable number to decrease the chances of Type 1 error. Covariates age and/or education were entered in the first step if they were significantly correlated with the dependent variable. All analyses are shown in Table 3. For the SDMT, although the group effect was statistically significant regardless of the ordering of the variables, the amount of variance accounted for by the group variable dropped from $10 \%$ to $6 \%$ when the MRR mean was entered before the group variable. For the combined PASAT, the amount of variance accounted for by the group variable declined from a statistically significant $4 \%$ of variance accounted for to a non-significant $2 \%$ when the MRR mean was entered before the group variable. Similarly with the COWAT, the amount of variance accounted for by the group variable declined from a statistically significant $5 \%$ to a non-significant $2 \%$ when the MRR mean was entered before the group variable. Finally, for the Animal Naming task, although the group variable was statistically significant regardless of the ordering of variables, the amount of variance accounted for by the group variable dropped from $11 \%$ to $7 \%$ when the MRR mean was entered before the group variable.

To examine discriminant validity, a comparable set of regression analyses were conducted on the cognitive measures not requiring a rapid oral response. For the CVLT-II, the group effect was statistically significant regardless of the ordering of the variables, with the amount of variance accounted for by the group variable dropping only from $6 \%$ to $5 \%$ when the MRR mean was entered before the group variable. For the BVMT-R, the group effect was again statistically significant regardless of the ordering of the variables, with the amount of variance accounted for by the group variable dropping only from $8 \%$ to $6 \%$ when the MRR mean was entered before the group variable.

To summarize, for speeded oral tasks, significant group effects for the COWAT and the PASAT were reduced to being non-significant when group differences in oral motor

\footnotetext{
${ }^{1}$ Because multicollinearity among predictors can be problematic in regression analyses, we evaluated a number of indicators of multicollinearity suggested by Maruyama (1997). Although these indicators did suggest some small relationship among our predictor variables, none met criterion for multicollinearity in any analysis.
}

speed were accounted for first; for Animal Naming and the SDMT, group effects were reduced by $36 \%$ (from $11 \%$ to $7 \%)$ and $40 \%(10 \%$ to $6 \%)$, respectively, when oral motor

Table 3. Regression analyses changing order of group variable

\begin{tabular}{|c|c|c|c|}
\hline & $\Delta \mathrm{r}^{2}$ & $\Delta \mathrm{F}$ & $p$ \\
\hline \multicolumn{4}{|c|}{ SDMT (Oral) } \\
\hline Step 1: Education, Age & .19 & 11.41 & $<.001$ \\
\hline Step 2: Group variable & .10 & 12.87 & $<.001$ \\
\hline Step 3: MRR mean & .07 & 9.58 & $<.005$ \\
\hline Step 1: Education, Age & .19 & 11.41 & $<.001$ \\
\hline Step 2: MRR mean & .10 & 13.91 & $<.001$ \\
\hline Step 3: Group variable & .06 & 8.59 & $<.005$ \\
\hline \multicolumn{4}{|c|}{ PASAT Combined } \\
\hline Step 1: Education, Age & .13 & 7.03 & $\leq .001$ \\
\hline Step 2: Group variable & .04 & 4.83 & $<.05$ \\
\hline Step 3: MRR mean & .04 & 4.40 & $<.05$ \\
\hline Step 1: Education, Age & .13 & 7.03 & $\leq .001$ \\
\hline Step 2: MRR mean & .06 & 6.41 & $<.05$ \\
\hline Step 3: Group variable & .02 & 2.87 & $<.10$ \\
\hline \multicolumn{4}{|c|}{ COWAT } \\
\hline Step 1: Group variable & .05 & 5.44 & $<.05$ \\
\hline Step 2: MRR mean & .09 & 9.79 & $<.005$ \\
\hline Step 1: MRR mean & .12 & 13.35 & $<.001$ \\
\hline Step 2: Group variable & .02 & 2.20 & $\mathrm{~ns}$ \\
\hline \multicolumn{4}{|c|}{ Animal Naming } \\
\hline Step 1: Age & .14 & 15.73 & $<.001$ \\
\hline Step 2: Group variable & .11 & 13.64 & $<.001$ \\
\hline Step 3: MRR mean & .03 & 4.38 & $<.05$ \\
\hline Step 1: Age & .14 & 15.73 & $<.001$ \\
\hline Step 2: MRR mean & .07 & 7.93 & $<.01$ \\
\hline Step 3: Group variable & .07 & 9.85 & $<.005$ \\
\hline \multicolumn{4}{|c|}{ CVLT-II } \\
\hline Step 1: Group variable & .06 & 6.20 & $<.02$ \\
\hline Step 2: MRR mean & .00 & $<1.00$ & ns \\
\hline Step 1: MRR mean & .00 & $<1.00$ & ns \\
\hline Step 2: Group variable & .05 & 5.35 & $<.05$ \\
\hline \multicolumn{4}{|c|}{ BVMT-R } \\
\hline Step 1: Education, Age & .28 & 18.49 & $<.001$ \\
\hline Step 2: Group variable & .08 & 11.78 & $<.002$ \\
\hline Step 3: MRR mean & .03 & 4.20 & $<.05$ \\
\hline Step 1: Education, Age & .28 & 18.49 & $<.001$ \\
\hline Step 2: MRR mean & .05 & 7.25 & $<.01$ \\
\hline Step 3: Group variable & .06 & 8.55 & $<.005$ \\
\hline
\end{tabular}

Note. MRR $=$ Maximum Repetition Rate of Syllables and Multisyllabic Combinations task (Kent et al., 1987) (syllables per second); PASAT = Paced Auditory Serial Addition Test (S.M. Rao, and the Cognitive Function Study Group of the National Multiple Sclerosis Society, 1990); COWAT $=$ Controlled Oral Word Association Test (Benton \& Hamsher, 1989); SDMT = Symbol Digit Modalities Test (A. Smith, 1982); CVLT$\mathrm{II}=$ California Verbal Learning Test, 2nd edition (Delis et al., 2000); BVMT-R = Brief Visual Memory Test-Revised (Benedict, 1997). 
speed differences were accounted for first. For the nonspeeded tasks, group differences remained statistically significant regardless of whether oral motor speed differences were accounted for first. Thus for these latter tasks, accounting for oral motor speed did not significantly impact the final group effect. With that said, oral motor speed did significantly predict performance on the BVMT-R, a finding that is inconsistent with our assumption that our oral motor speed measure only reflects simple motor speed.

A final set of analyses were conducted to evaluate possible factors responsible for MS patients' slower oral motor speed. Because numerous studies have shown that individuals with MS are characterized by greater depression (Goldman Consensus Group, 2005) and fatigue (MacAllister \& Krupp, 2005) than healthy controls, and because both depression and fatigue might be expected to cause psychomotor slowing, both variables were examined in relation to oral motor speed. The mean MRR score was significantly correlated with both the FSS $(r(100)=-.27, p<.01)$ and the BDI-II $(r(100)=-.32, p \leq .001)$. Two covariance analyses (ANCOVA) were conducted. Both included the Control-MS group as the between groups factor, with the BDI-II used as the covariate in one analysis and the FSS used as the covariate in the other. The ANOVA conducted without the covariates resulted in a significant group effect $(F(1,98)=7.95, p<.01)$. When the ANCOVA was conducted with FSS as the covariate, this group effect was reduced to being non-statistically significant $(F(1,97)=$ $1.33, n s)$. A similar result was found when the BDI-II was used as a covariate $(F(1,97)=1.92, n s)$.

\section{DISCUSSION}

The results of this study show that individuals with MS display objective articulation speed deficits, which contribute to performance on commonly used neuropsychological tests. More specifically, the amount of variance accounted for by the group (MS-Control) variable in regression analyses was reduced the following amounts for the tasks when the mean oral motor speed variable was entered before the group variable: SDMT, $10 \%$ to $6 \%$; PASAT, $4 \%$ to $2 \%$; COWAT, $5 \%$ to $2 \%$; Animal Naming, $11 \%$ to $7 \%$. Though group effects for the SDMT and Animal Naming tests remained significant $(p<.05)$ when the MRR score was entered first, group variance accounted for was reduced by over a third, whereas the group effects for both the PASAT and COWAT were reduced from being significant to non-significant.

Although a consensus statement on measuring cognitive functioning in MS (Benedict et al., 2002) recommended the use of the MRR task when the MACFIMS is administered to MS patients, this study provides the first empirical evidence that this task is associated with poor performance on NP tests that require an oral response. Thus, failure to control for rudimentary oral motor problems in neuropsychological evaluations of these patients is likely to present an inflated picture of their cognitive deficits. Neuropsycholog- ists are trained to adjust performance expectations on manual tasks in patients with upper extremity signs, such as mild weakness, tremor, or evidence of poor hand coordination. Our data suggest that the same is true for oral articulation defects and the MRR, at least in the case of MS. Further research on the development of more sensitive measures of information processing speed in MS that provide more systematic control for the slow oral motor speed articulation problems common to many individuals with MS appears warranted.

Another important finding from our study is that, although group variance accounted for in the SDMT and Animal Naming tasks was reduced by over a third when basic oral motor speed was considered first, differences with healthy controls remained statistically significant. Additionally, both tasks initially accounted for much more group variance than either the PASAT or COWAT, suggesting that the SDMT and Animal Naming are not only more sensitive to cognitive dysfunction in MS compared with these other two tasks, but are less sensitive to the effects of impairments in oral motor speed. That said, our finding that Animal Naming was more sensitive than the COWAT to cognitive problems in MS patients is inconsistent with a recent meta-analysis suggesting that the tests are equally sensitive (Henry \& Beatty, 2006).

An interesting finding to emerge from our study was that group differences in fatigue and depression accounted for the group differences observed in oral motor speed. This is not surprising given that psychomotor slowing is a core symptom of depression and fatigue. If depression and fatigue are causally related to slowed oral motor speed, then successful treatment of these common features of MS might result in improved oral motor speed and NP testing more generally. A treatment intervention study of fatigue or depression in MS would help to tease out the possible causal relationships between these variables. If slowed oral motor speed in MS was partially reversible by such intervention, it might lead to better perceived cognitive functioning in these patients and better actual cognitive performance.

Although it is tempting to speculate that the oral motor speed problems in our patients with MS are reversible and a function of such factors as depression and fatigue, it is also possible that slowed speech in these patients is a marker for the level of neuropathology present and is thus correlated with cognitive test performance because of this third variable association. It has already been consistently established that cognitive problems in MS are associated with the extent of lesion damage in the brain (Arnett, 2003; Rao et al., 1989), gray matter hypointensities (Brass et al., 2006), and especially atrophy (Benedict et al., 2006). It may be that slowed speech in MS is comparably related to such brain damage. Related to this issue, we did find that the MRR was significantly associated with the BVMT-R, a task with no oral motor demands but one which is highly sensitive to MS pathology (Benedict et al., 2006). This finding suggests the possibility that oral motor deficits in MS may also be a marker for neuropsychological compromise. How- 
ever, inconsistent with this conceptualization is the fact that performance on another neuropsychological task not requiring a rapid oral response, the CVLT-II, was not associated with MRR performance. It may be that the BVMT-R, despite having no oral motor demands, was significantly associated with the MRR because of the task's motor drawing component. This would suggest that the MRR may generally index motor system dysfunction. These speculations aside, follow-up research could examine the association of the MRR with more direct measures of brain damage in the context of the same types of cognitive tests used in the current study to help clarify these interpretive issues.

Our data may also have implications for the assessment of cognitive functioning in other neurological patient groups where dysarthria is common (e.g., Parkinson's disease, traumatic brain injury). It may be that, like MS patients, estimates of cognitive problems in such patient groups are inflated by their slowed speech. Follow-up research in these populations employing paradigms similar to those used in the current study would provide a first step in addressing this possibility.

Our study is not without limitations. First, our MS and control groups were not well matched on either age or education, with controls significantly younger and more educated than our MS participants. Although we controlled for such differences statistically in our analyses, a more rigorous methodological approach would have been to have our groups matched to begin with, eliminating the need for statistical correction. Second, our sample size was relatively small; however, given the significant group differences we observed in both neuropsychological and oral motor test performance, we had adequate statistical power to detect hypothesized effects.

It has long been known that both input and output problems could influence performance on neuropsychological tests (Weintraub \& Mesulam, 1985). In neuropsychology, we hope to draw conclusions about the nature of cognitive difficulties with our tests. It is not possible to do this, however, without first ruling out that input or output channels might be impaired. Difficulty perceiving test stimuli accurately (input problem) or problems in response output systems (typically written or spoken response) can undoubtedly affect performance on many commonly used neuropsychological tests and make it difficult to draw conclusions relating specifically to cognitive dysfunction. Various ingenious strategies have been developed by clinical and experimental neuropsychologists over the years to address these problems. In the MS literature, given MS patients' well known difficulties with fine and gross motor coordination and speed, a typical approach to address output problems has been to eliminate tests requiring manual motor or written responses. Our data suggest, however, that eliminating such tests and relying upon measures requiring only a spoken output does not completely resolve this issue. It appears that the slowed verbal output of MS patients also interferes with optimal neuropsychological task performance, making clear conclusions specific to cognitive processes difficult. Given this, it is incumbent upon neuropsychologists to be aware of this influence and, ultimately, to develop tasks that allow for a more systematic control of oral motor slowing so that a clearer picture of the nature of the cognitive difficulties characterizing MS patients can emerge. Future research will be necessary to explore these issues in more detail, in both MS patients and other patient groups characterized by slowed speech.

\section{ACKNOWLEDGMENTS}

This investigation was supported (in part) by a grant to the first author from the National Multiple Sclerosis Society (PP0978). The authors thank the many neurologists in the Pennsylvania region who contributed their time to verifying MS diagnoses and ratings of course for the MS participants in the project. The authors also thank Andrae Laws and Joseph Beeney for their help with various aspects of the study.

\section{REFERENCES}

Amato, M.P., Zipoli, V., \& Portaccio, E. (2006). Multiple sclerosisrelated cognitive changes: A review of cross-sectional and longitudinal studies. Journal of the Neurological Sciences, 245, 41-46.

Arnett, P.A. (2003). Neuropsychological presentation and treatment of demyelinating disorders. In P. Halligan, U. Kischka, \& J. Marshall (Eds.), Handbook of Clinical Neuropsychology (pp. 528-543). Oxford: Oxford University Press.

Arnett, P.A., Higginson, C.I., Voss, W.D., Wright, B., Bender, W.I., Wurst, J.M., \& Tippin, J.M. (1999). Depressed mood in multiple sclerosis: Relationship to capacity-demanding memory and attentional functioning. Neuropsychology, 13, 434-446.

Beck, A.T., Steer, R.A., \& Brown, G.K. (1996). Beck Depression Inventory Manual (3rd ed.). San Antonio, TX: The Psychological Corporation.

Benedict, R.H.B. (1997). Brief Visuospatial Memory Test-Revised: Professional Manual. Odessa, FL: Psychological Assessment Resources.

Benedict, R.H.B., Bruce, J.M., Dwyer, M.G., Abdelrahman, N., Hussein, S., Weinstock-Guttman, B., Garg, N., Munschauer, F., \& Zivadinov, R. (2006). Neocortical atrophy, third ventricular width, and cognitive dysfunction in multiple sclerosis. Archives of Neurology, 63, 1301-1306.

Benedict, R.H.B., Fischer, J.S., Archibald, C.J., Arnett, P.A., Beatty, W.W., Bobholz, J., Chelune, G.J., Fisk, J.D., Langdon, D.A., Caruso, L., Foley, F., LaRocca, N.G., Vowels, L., Weinstein, A., DeLuca, J., Rao, S.M., \& Munschauer, F. (2002). Minimal neuropsychological assessment of MS patients: A consensus approach. The Clinical Neuropsychologist, 16, 381-397.

Benton, A.L. \& Hamsher, K.deS. (1989). Multilingual Aphasia Examination. Iowa City, IA: AJA Associates.

Brass, S.D., Benedict, R.H.B., Weinstock-Guttman, B., Munschauer, F., \& Bakshi, R. (2006). Cognitive impairment is associated with subcortical magnetic resonance imaging grey matter T2 hypointensity in multiple sclerosis. Multiple Sclerosis, 12, 437-444.

Brassington, J.C. \& Marsh, N.V. (1998). Neuropsychological aspects of multiple sclerosis. Neuropsychology Review, 8, 43-77. 
Charcot, J.M. (1877). Lectures on the diseases of the nervous system (G. Sigerson, Trans. Vol. 1): London: New Sydenham Society.

Darley, F.L., Brown, J.R., \& Goldstein, N.P. (1972). Dysarthria in multiple sclerosis. Journal of Speech and Hearing Research, 15, 229-245.

Delis, D.C., Kramer, J.H., Kaplan, E., \& Ober, B.A. (2000). California Verbal Learning Test manual: Second edition, adult version. San Antonio, TX: The Psychological Corporation.

Dikmen, S.S., Machamer, J.E., Winn, H.R., \& Temkin, N.R. (1995). Neuropsychological outcome at 1-year post head injury. Neuropsychology, 9, 80-90.

Dworkin, J.P., Aronson, A.E., \& Mulder, D.W. (1980). Tongue force in normals and in dysarthric patients with amyotrophic lateral sclerosis. Journal of Speech and Hearing Research, 23, 828-837.

Fischer, J.S., Foley, F.W., Aikens, J.E., Ericson, D.G., Rao, S.M., $\&$ Shindell, S. (1994). What do we really know about cognitive dysfunction, affective disorders, and stress in multiple sclerosis? A practitioner's guide. Journal of Neurological Rehabilitation, 8, 151-164.

Fischer, J.S., Rudick, R.A., Cutter, G.A., \& Reingold, S.C. (1999). The Multiple Sclerosis Functional Composite (MSFC): An integrated approach to MS clinical outcome assessment. Multiple Sclerosis, 3, 244-250.

Goldman Consensus Group. (2005). The Goldman Consensus statement on depression in multiple sclerosis. Multiple Sclerosis, $11,328-337$.

Hartelius, L., Runmarker, B., \& Andersen, O. (2000a). Prevalence and characteristics of dysarthria in a multiple-sclerosis incidence cohort: Relation to neurological data. Folia Phoniatrica et Logopedica, 52, 160-177.

Hartelius, L., Runmarker, B., Andersen, O., \& Nord, L. (2000b). Temporal speech characteristics of individuals with multiple sclerosis and ataxia dysarthria: "Scanning speech" revisited. Folia Phoniatrica et Logopedica, 52, 228-238.

Henry, J.D. \& Beatty, W.W. (2006). Verbal fluency deficits in multiple sclerosis. Neuropsychologia, 44, 1166-1174.

Jonsson, A., Andresen, J., Storr, L., Tscherning, T., Sorensen, P.S., \& Ravnborg, M. (2006). Cognitive impairment in newly diagnosed multiple sclerosis patients: A 4-year follow-up study. Journal of the Neurological Sciences, 245, 77-85.

Kent, R.D., Kent, J.F., \& Rosenbek, J.C. (1987). Maximum performance tests of speech production. Journal of Speech and Hearing Disorders, 52, 367-387.

Kreul, E.J. (1972). Neuromuscular control examination (NMC) for Parkinsonism: Vowel prolongations and diadochokinetic and reading rates. Journal of Speech and Hearing Research, $15,72-83$.

Krupp, L.B., LaRocca, N.G., Muir-Nash, J., \& Steinberg, A.D. (1989). The Fatigue Severity Scale: Application to patients with multiple sclerosis and systemic lupus erythematosus. Archives of Neurology, 46, 1121-1123.

Kurtzke, J.F. (1983). Rating neurologic impairment in multiple sclerosis: An Expanded Disability Status Scale (EDSS). Neurology, 33, 1444-1452.

Lezak, M.D., Howieson, D.B., \& Loring, D.W. (2005). Neuropsychological Assessment (4th ed.). New York: Oxford University Press.
Lublin, F.D. \& Reingold, S.C. (1996). Defining the clinical course of multiple sclerosis: Results of an international survey. $\mathrm{Neu}$ rology, 46, 907-911.

MacAllister, W.S. \& Krupp, L.S. (2005). Multiple sclerosisrelated fatigue. Physical Medicine and Rehabilitation Clinics of North America, 16, 483-502.

Maruyama, G.M. (1997). Basics of structural equation modeling. Thousand Oaks, CA: Sage Publications.

McDonald, W.I., Compston, A., Edan, G., Goodkin, D., Hartung, H.-P., Lublin, F.D., et al. (2001). Recommended diagnostic criteria for multiple sclerosis: Guidelines from the international panel on the diagnosis of multiple sclerosis. Annals of Neurology, 50, 121-127.

Portnoy, R. \& Aronson, A. (1982). Diadochokinetic syllable rate and irregularity in normal and in spastic and ataxic dysarthric subjects. Journal of Speech and Hearing Disorders, 47, 324-328.

Rao, S.M. \& The Cognitive Function Study Group of the National Multiple Sclerosis Society. (1990). Manual for the Brief Repeatable Battery of Neuropsychological Tests in Multiple Sclerosis. New York: National Multiple Sclerosis Society.

Rao, S.M., Leo, G.J., Bernardin, L., \& Unverzagt, F. (1991). Cognitive dysfunction in multiple sclerosis. 1 . Frequency, patterns, and prediction. Neurology, 41, 685-691.

Rao, S.M., Leo, G.J., Haughton, V.M., St. Aubin-Faubert, P., \& Bernardin, L. (1989). Correlation of magnetic resonance imaging with neuropsychological testing in multiple sclerosis. $\mathrm{Neu}$ rology, 39, 161-166.

Robertson, I.H., Ward, T., Ridgeway, V., \& Nimmo-Smith, I. (1994). The Test of Everyday Attention. Suffolk, England: Thames Valley Testing Co.

Smith, A. (1982). Symbol Digit Modalities Test (SDMT) Manual (Revised). Los Angeles: Western Psychological Services.

Smith, M.M. \& Arnett, P.A. (2007). Dysarthria predicts poorer performance on cognitive tasks requiring a speeded oral response in an MS population. Journal of Clinical and Experimental Neuropsychology, 29, 804-812.

Strauss, E., Sherman, E.M.S., \& Spreen, O. (2006). A compendium of neuropsychological tests (3rd ed.). New York, NY: Oxford University Press.

Tatsumi, I., Sasanuma, S., Hirose, H., \& Kirtani, S. (1979). Acoustic properties of ataxic and parkinsonian speech in syllable repetition tasks. Annual Bulletin of the Royal Institute of Logopedics and Phoniatrics (Tokyo), 13, 99-104.

Tröster, A.I. \& Arnett, P.A. (2006). Assessment of movement and demyelinating disorders. In P.J. Snyder, P.D. Nussbaum, \& D.L. Robins (Eds.), Clinical neuropsychology: A pocket handbook for assessment (2nd ed.), (pp. 243-293). Washington, DC: The American Psychological Association.

Wechsler, D. (1997). Wechsler Adult Intelligence Scale (3rd ed.). San Antonio, TX: The Psychological Corporation.

Weintraub, S. \& Mesulam, M.-M. (1985). Mental state assessment of young and elderly adults in behavioral neurology. In M.-M. Mesulam (Ed.), Principles of Behavioral Neurology: F.A. Davis Co.

Ziegler, W. \& Wessel, K. (1996). Speech timing in ataxic disorders: Sentence production and rapid repetitive articulation. Neurology, 47, 208-214. 